\title{
Influence of Protection on Transient Stability of Medium Voltage Grids including Distributed Generation
}

E.J. Coster ${ }^{(1,2)}$, J.M.A. Myrzik ${ }^{(2)}$, W.L. Kling ${ }^{(2)}$

(1) ENECO NetBeheer, the Netherlands, (2) Eindhoven University of Technology, the Netherlands

\begin{abstract}
Due to the increase of distributed generation (DG) in the future it can become important to keep DG connected to the grid in order to maintain the balance between consumed and generated electrical power. Keeping DG-units connected to the grid during a disturbance, the dynamic behavior of the DG-units becomes important. Protection schemes based on definite and inverse over-current are simulated and transient stability of DG-units is investigated. The simulations have shown that there is no major benefit to apply inverse over-current relays in MV-grids with short feeder length and high short circuit power. With conventional grading of the protection in most cases the DG-units become unstable. The paper shows that reduction of the time grading is possible however, this reduction is not sufficient to guarantee stable operation. The best option is fast fault clearing of the complete feeder. It is shown that this is possible without loss of selectivity and with stable operation of the remaining DG-units.
\end{abstract}

Keywords: Transient Stability, Protection, Combined Heat and Power, Medium Voltage Grid

\section{INTRODUCTION}

At the moment the amount of distributed generation (DG) connected to the medium voltage grid (MV-grid) is increasing. In certain parts of the Netherlands this growth is due to the development of greenhouse areas. In each greenhouse a small Combined Heat and Power plant (CHP) is installed which consists of a synchronous generator and a natural gas engine as prime mover. Besides electricity the CHP-plant also produces heat and $\mathrm{CO}_{2}$ which is used in the greenhouse and the electricity is sold to the market.

Most of the MV-grids have a simple radial topology. This radial structure allows simple protection schemes. Connection of DG to MV-grids can affect proper operation of the protection schemes. According to various international standards [1] as well as national grid codes, during a disturbance DG-units have to be disconnected immediately. In this way DG units do not contribute to the continuous short circuit current and proper operation of the protection system is guaranteed. In future with a larger amount of DG it will become important to keep the generators connected to the grid $[2,3]$ in order to maintain balance between generation and load.

In this paper the effect of the grid protection on the transient stability of MV-grids including CHP-plants will be discussed. This will be done by studying the current situation with an under-voltage protection of the CHPplants. This is the base case and a comparison will be made for the case when the under-voltage protection of the CHP-plants is omitted. Omitting the under-voltage protection prevents immediate disconnection of the CHPplants and offers the possibility to study the dynamic behaviour of the CHP-plants. For the CHP-plants the Critical Clearing Time (CCT) will be calculated and the effect of the settings of the network protection will be determined. Protection schemes including definite overcurrent protection and inverse over-current protection will be simulated. The paper ends with a discussion on the influence of these protection schemes on the transient stability of MV-grids.

\section{TEST GRID}

In this section the connection scheme of DG-units to the MV-grid is described. The test grid used in this paper is an existing $20 \mathrm{kV}$ MV-grid. The general grid lay out is shown in figure 1. The MV-grid is located in a greenhouse area and is characterised by a short feeder length between the connections of the separate greenhouses $(0.1<l<1.5 \mathrm{~km})$. The HV/MV substation contains three HV/MV transformers and is build outside the area which results in relative long feeder length to the greenhouse area $(l>5$ $\mathrm{km})$. The MV-grid has a ring structure but is operated radial. In figure 1 the grid openings are indicated.

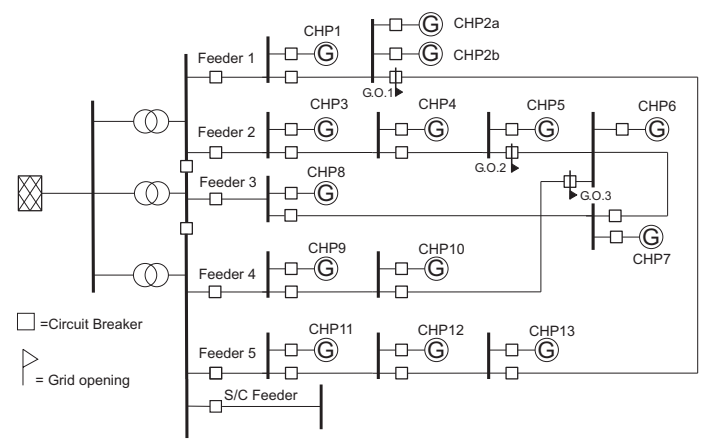

\section{Figure 1 Overview of $20 \mathrm{kV}$ test grid}

To the MV-grid 14 DG-units are connected. Except CHP 2a, 4, 5 and 8 all units have an apparent power of 3 MVA, p.f 0.8. CHP 2a, 4,5 and 8 have a capacity of 1.5 MVA, p.f. 0.8. All units are operated with unity power factor. For the cable connections XLPE $3 \times 1 \times 630 \mathrm{~mm}^{2} \mathrm{Al}$ cable is used. In the grid an additional short circuit feeder (S/Cfeeder) is added which is used in the simulations as faulted feeder.

In figure 2 an overview of a typical MV-grid connection scheme of DG-units including the generator protection is shown. Due to the capacity of DG-units, greenhouses have a connection to MV-grids by a $20 \mathrm{kV}$ Ring Main Unit (RMU). In this RMU, connection cubicles for DG and load are present as well as cubicles for grid connection. The protection of the DG will trip the accompanying 
circuit breaker. In figure 2 only the protection which have an interaction with the grid are considered and the typical generator protection is omitted. In the figure the IEEE/ANSI designated numbers are used. In the test grid all DG-units are modeled conform figure 2.

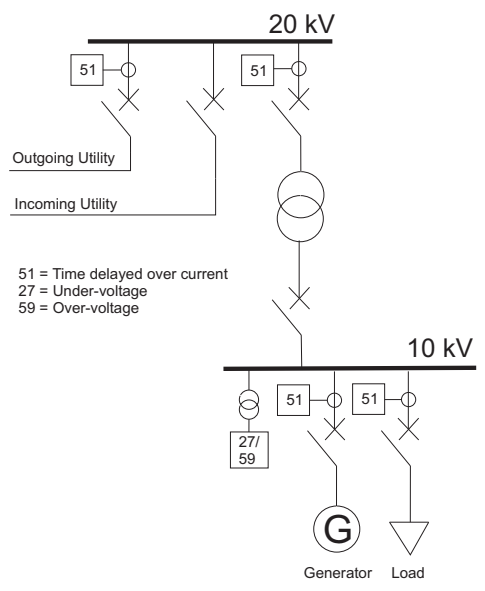

Figure 2 Overview of a typical connection of DG to the MV-grid

\section{CURRENT PROTECTION SCHEME}

The current protection scheme is based on definite overcurrent protection. The protection relays are located at the outgoing feeders in the substation. The settings of the relays are based on short circuit calculations and time grading is done is such a way that selectivity with the connected customer is encountered. In figure 3 the settings of the current protection scheme are depicted.

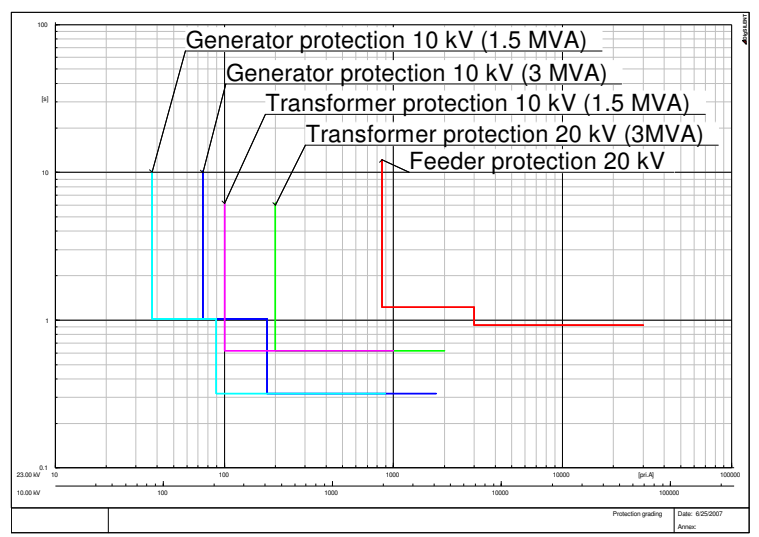

Figure 3 Time-over-current plot current protecting scheme

Besides over-current protection the generator is equipped with an under-voltage protection which is set at 0.8 p.u.$200 \mathrm{~ms}$. The protection scheme is implemented in the test grid and simulations are carried out to test the protection scheme. Therefore a three phase short circuit is made at the S/C-feeder (see figure 1). In this way each generator suffers the same kind of disturbance. The result of the simulation is depicted in table 1 . The simulation shows that all DG-units are disconnected by the under-voltage protection before the fault in the S/C-feeder is cleared.
Table 1: Reaction of the protection scheme

\begin{tabular}{|l|l|l|l|}
\hline & \multicolumn{3}{|c|}{ Protection } \\
\hline Component & $\mathrm{U}<$ & $\mathrm{I}>>$ & Fault Clearing time \\
\hline Generator & $0.2 \mathrm{~s}$ & $0.3 \mathrm{~s}$ & $0.2 \mathrm{~s}$ \\
\hline Step-up transformer & -- & $0.6 \mathrm{~s}^{1}$ & -- \\
\hline Feeders & -- & -- & -- \\
\hline S/C feeder & -- & $0.9 \mathrm{~s}$ & $0.9 \mathrm{~s}$ \\
\hline
\end{tabular}

\section{STABILITY OF DG-UNITS}

As mentioned earlier a grid disturbance in the MV-grid leads to a massive disconnection of DG due to the undervoltage protection. In the future it can become important to keep the DG-units connected to the grid in order to keep the power balance. This is expressed in $[2,3]$. This can be reached by changing the protection scheme and settings. Keeping the DG-unit connected to the grid during a disturbance may lead to instability of the synchronous generator. The behavior of DG-units during a disturbance can be described with the well known swing equation [4].

$$
\frac{2 H}{\omega_{s}} \frac{d^{2} \delta}{d t^{2}}=P_{m}-P_{e}
$$

With the electrical delivered power to the grid:

$$
P_{e}=\frac{E \cdot U}{X} \sin \delta
$$

With the aid of the equal area criterion for the critical clearing time (CCT) can be derived:

$$
t_{c r}=\sqrt{\frac{4 H\left(\delta_{c r}-\delta_{0}\right)}{\omega_{s} P_{m}}}
$$

A detailed description and derivation can be found in [5]. In equation (1)-(3) $\mathrm{H}$ is the inertia constant, $\mathrm{P}_{\mathrm{m}}$ the mechanical power, $\omega_{\mathrm{s}}$ the synchronous rotor speed, $\delta_{\mathrm{cr}}$ the critical clearing angle and $\delta_{0}$ the initial rotor angle. The mentioned equations are principally only valid for two machine systems. However, the CCT of the DG-units determined by simulation shows a great similarity with the values calculated with (3). In table 2 the critical clearing time for both the DG sizes connected in the test grid is given. These values are in agreement with values found in literature [2].

Table 2: Critical Clearing Time (CCT)

\begin{tabular}{|c|c|c|c|c|}
\hline $\mathrm{S}_{\text {gen }}[\mathrm{MVA}]$ & $\mathrm{P}_{\text {gen }}[\mathrm{MW}]$ & $\mathrm{H}[\mathrm{s}]$ & $\mathrm{t}_{\mathrm{cr}, \text { calc }}[\mathrm{ms}]$ & $\mathrm{t}_{\mathrm{cr}, \text { sim }}[\mathrm{ms}]$ \\
\hline 3 & 2.4 & 1.7 & 225 & 230 \\
\hline 1.5 & 1.2 & 1.3 & 220 & 215 \\
\hline
\end{tabular}

Equation (2) holds for high voltage grids with $X>>R$. In $\mathrm{MV}$-grids $\mathrm{X} \approx \mathrm{R}$, hence the grid resistance cannot be neglected anymore. When $\mathrm{X}$ is increasing, the coupling of the generator to the grid becomes weaker which results in a shorter CCT. In MV-grids this effect is not observed as mentioned below. The CCTs shown in table 2 are based on a three phase short circuit at the terminals of the generator. In comparison with these values, simulations show an

\footnotetext{
${ }^{1} \mathrm{~S} / \mathrm{C}$ contribution sensed by protection without fault clearing
} 
increase of CCT for disturbances at different locations in the grid. The results of the simulation of a terminal short circuit of CHP 1 and the results of the simulation of a three phase fault at the end of S/C-feeder are depicted in figure 4. In the first case the terminal voltage of CHP 1 is zero and no electric power is delivered to the grid. In the second case a small amount of power is delivered to the grid. In the test grid no load is connected, hence the delivered power by the generators can only be consumed by the resistance in the grid.

During the disturbance at the S/C-feeder the generator is delivering power, $\mathrm{P}_{\mathrm{e}}$, and according to (1) the increase of the rotor angle is limited with respect to the increment of the rotor angle during the terminal short circuit. This is also shown in figure 4 . Due to the power consumption by the grid resistance stability of the DG-units is improved.

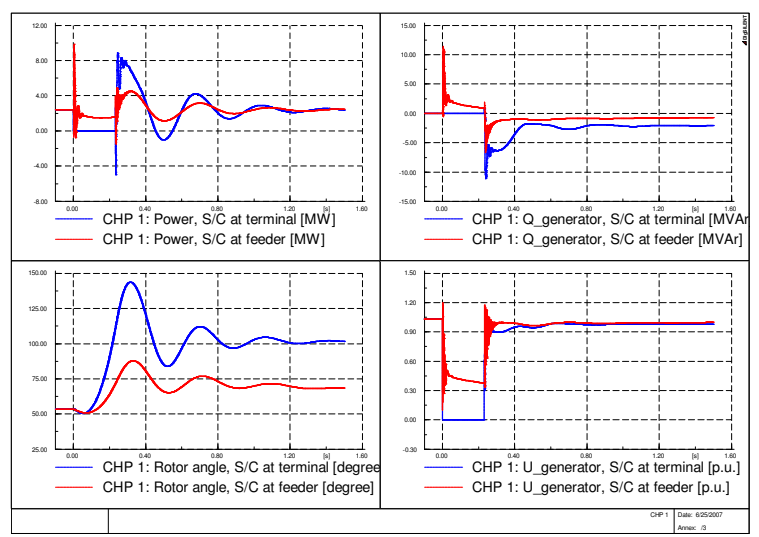

Figure 4 Effect of grid resistance on CCT

\section{AVERAGE CLEARING TIME}

To compare the performance of protection schemes the average clearing time is introduced. In [6] this parameter is only indicated without calculation, however, here the parameter is calculated in detail.

The S/C-current in a feeder is dependent on the grid and feeder impedance as well as the location of the disturbance. The S/C-current as function of the distance is:

$$
I_{k}^{\prime \prime}(l)=\frac{U}{\sqrt{3}\left|Z_{\text {grid }}+z \cdot l\right|}
$$

In (4) $\mathrm{z}$ is the complex feeder impedance per unit length, $\mathrm{Z}_{\text {grid }}$ is the complex grid impedance and $\mathrm{U}$ the line voltage. In general for the fault clearing time of an inverse overcurrent relay holds [8]:

$$
t_{\text {clear }}\left(I_{k}^{\prime \prime}\right)=\frac{\beta \cdot T_{p}}{\left(\frac{I_{k}^{\prime \prime}}{I_{p}}\right)^{\alpha}-1}
$$

The characteristic of the relay is determined by the parameters $\alpha$ and $\beta$ which are for a normal inverse characteristic 0.02 and 0.14 [7]. $\mathrm{T}_{\mathrm{p}}$ is the grading parameter and $I_{p}$ the pick-up current. The clearing time $t_{\text {clear }}$ is a function of the short circuit current $\mathrm{I}_{\mathrm{S} / \mathrm{C}}$. The fault clearing time as function of the feeder length is obtained by combining (4) and (5) and shown in (6).

$$
t_{\text {clear }, m}=\frac{\beta \cdot T_{p}}{\left(\frac{U}{\sqrt{3}\left|Z_{\text {grid }}+z \cdot l\right| \cdot I_{p}}\right)^{\alpha}-1}
$$

For a feeder with $n$ sections, for section $m$ the average clearing time is:

$$
t_{a v, m}=\frac{1}{l_{m+1}-l_{m}} \int_{m}^{m+1} t_{c l e a r, m}(l) d l
$$

Equation (7) cannot be solved analytically hence to solve this equation numerical integration methods are applied. The average clearing time for a feeder of $n$ sections can be calculated with:

$$
t_{a v}=\frac{1}{n} \sum_{m=1}^{n} t_{a v, m}
$$

The average clearing time is also affected by the applied time grading. To avoid loss of selectivity the applied time grading is between $0.3-0.5 \mathrm{~s}$ [8]. The time grading consists of the following components:

$$
t_{\text {grading }}=t_{\text {tol }}+t_{\text {delay }}+t_{\text {drop }}+t_{\text {C.B. }}
$$

In (9) $t_{\text {tol }}$ is the tolerance of the relay, $t_{\text {delay }}$ is the pick-up time of the relay, $t_{\text {drop }}$ is the drop-off time of the relay and $t_{\text {C.B. }}$ is the switching time of the circuit breaker. However, the mentioned time grading is based on electromechanical relays which are slower than the modern digital relays. In MV-grids including RMUs with vacuum breakers and digital relays, the time grading can be reduced. In table 3 for a modern circuit breaker and digital protection relay the separate components of (9) are shown [7].

Table 3: Data of digital relay and vacuum breaker

\begin{tabular}{|c|c|c|c|c|}
\hline $\mathrm{t}_{\text {tol }}[\mathrm{ms}]$ & $\mathrm{t}_{\text {delay }}[\mathrm{ms}]$ & $\mathrm{t}_{\text {drop }}[\mathrm{ms}]$ & $\mathrm{t}_{\mathrm{C} . \mathrm{B} .}[\mathrm{ms}]$ & $\mathrm{t}_{\text {grading }}[\mathrm{ms}]$ \\
\hline 10 & 30 & 35 & 50 & 125 \\
\hline
\end{tabular}

In the next section the current and the proposed time grading is applied and the effect on transient stability of the CHP plants is discussed.

\section{EVALUATION OF PROTECTION SCHEMES}

In this section the influence of various protection schemes and settings on stability of DG-units are discussed. To demonstrate the effect of the protection schemes, feeder 1 of the test grid is used. Therefore grid opening 1 (G.O.1) is closed and the breaker of feeder 5 is opened. In this way a feeder including 5 RMUs and 6 generators is created. In figure 5 a schematic diagram of feeder 1 including protection is depicted. On this feeder two protection schemes are evaluated:

1. Protection of each RMU by a definite overcurrent relay

2. Protection of each RMU by an inverse overcurrent relay 


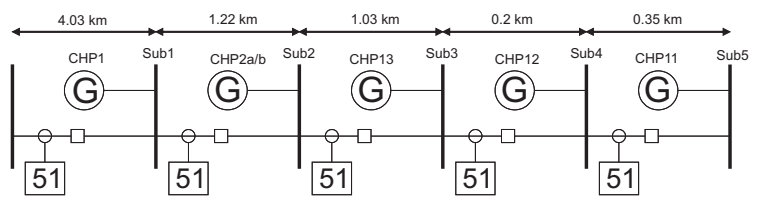

Figure 5 Schematic overview of feeder 1 including protection

To guarantee selectivity for both protection schemes traditional grading of $0.3 \mathrm{~s}$ is applied. The pick-up value of the definite over-current relays is based on $50 \%$ of the three phase S/C-current at the point of connection of the relay [8]. The S/C-current is calculated without the contribution of the CHP plants and is approximately $9 \mathrm{kA}$. For the setting of the inverse over-current relay the TAP and DIAL parameter have to be determined. The TAP value is obtained by applying (10) [8].

$$
T A P=\frac{1,5 \cdot I_{\text {nom }}}{C T R}
$$

In (10) $I_{\text {nom }}$ is the nominal current of the protected feeder and CTR is the CT transformation ratio. The DIAL setting, $\mathrm{T}_{\mathrm{p}}$, is determined by:

$$
T_{p, n+1}=T_{p, n}+\frac{t_{\text {grading }}}{\beta}\left[\left(\frac{I_{k}^{\prime \prime}}{I_{p}}\right)^{\alpha}-1\right]
$$

Where $T_{p, n}$ is the DIAL setting of the relay at location $n$ and $\mathrm{T}_{\mathrm{p}, \mathrm{n}+1}$ is the DIAL setting of the relay at the first upstream location. The lowest DIAL setting is assigned to the relay which is the furthest away from the source. The DIAL settings are calculated for a normal inverse characteristic $(\alpha=0.02$ and $\beta=0.14)$ and $t_{\text {grading }}=0.3 \mathrm{~s}$. In table 4 an overview of the relay settings of both protection schemes is given.

Table 4: Overview of protection settings

\begin{tabular}{|c|c|c|c|c|c|}
\hline & \multirow{2}{*}{$\begin{array}{c}\text { CT- } \\
\text { Ratio }\end{array}$} & \multicolumn{2}{|c|}{ Definite } & \multicolumn{2}{c|}{ Inverse } \\
\cline { 3 - 6 } & $\mathrm{I} \gg$ & $\mathrm{t} \gg>$ & $\mathrm{T}_{\mathrm{p}}$ & $\mathrm{I}_{\mathrm{p}}$ \\
\hline Sub 1 & $750 / 5 \mathrm{~A}$ & $4500 \mathrm{~A}$ & $1,3 \mathrm{~s}$ & 0.43 & 7.1 \\
\hline Sub 2 & $750 / 5 \mathrm{~A}$ & $4500 \mathrm{~A}$ & $1,0 \mathrm{~s}$ & 0.33 & 7.1 \\
\hline Sub 3 & $750 / 5 \mathrm{~A}$ & $4500 \mathrm{~A}$ & $0,7 \mathrm{~s}$ & 0.24 & 7.1 \\
\hline Sub 4 & $750 / 5 \mathrm{~A}$ & $4500 \mathrm{~A}$ & $0,4 \mathrm{~s}$ & 0.14 & 7.1 \\
\hline Sub 5 & $750 / 5 \mathrm{~A}$ & $4500 \mathrm{~A}$ & $0,1 \mathrm{~s}$ & 0.05 & 7.1 \\
\hline
\end{tabular}

To compare the performance of the protection schemes, with the aid of (6)-(8) for both cases the average clearing time is calculated. Tripping time of a definite over-current protection scheme is independent of the level of fault current. Hence for the definite case $t_{p, m}$ is constant and (7) yields just the time setting of the relay. In this case the average clearing time can be calculated by summing up the time settings of the relays divided by the number of relays. For the given settings both protection schemes have an average clearing time of $0.7 \mathrm{~s}$. The average clearing time exceeds the CCT of the connected DG-units. Simulation of short circuits at the substations of feeder 1 both protection schemes show that all disturbances are cleared properly, however, stable operation of the DGunits is only guaranteed for disturbances between substation 4 and 5. Comparison of the protection schemes lead to the conclusion that for feeder 1 the inverse overcurrent protection yields no extra benefit versus the definite over-current protection.

Due to the contribution of DG-units to the short circuit current, the sensed short circuit current by relay $n$ downstream the generator is increased and the sensed short circuit current by relay $\mathrm{n}+1$ upstream the generator is decreased. When inverse over-current relays are applied, this effect leads to an improved selectivity [6]. Simulations are carried out to investigate the influence of the contribution of the DG-units to the short circuit current on the fault clearing time. Therefore in feeder 1 a disturbance at every substation is simulated with and without the influence of the DG-units. The results are presented in table 5 .

Table 5: Effect of DG on fault clearing time

\begin{tabular}{|l|c|c|}
\hline \multirow{2}{*}{ Fault location } & \multicolumn{2}{|c|}{$\mathrm{t}_{\text {clear }}[\mathrm{ms}]$} \\
\cline { 2 - 3 } & With DG & Without DG \\
\hline Sub 5 & 170 & 176 \\
\hline Sub 4 & 468 & 471 \\
\hline Sub 3 & 792 & 800 \\
\hline Sub 2 & 1000 & 1066 \\
\hline Sub 1 & 1323 & 1330 \\
\hline
\end{tabular}

Due to short feeder length and high short circuit power of the grid the contribution of the DG-units to the short circuit current are marginal and as well as the effect on fault clearing time.

As discussed earlier by applying modern digital relays the time grading can be reduced without loss of selectivity. To determine the effect of the reduction of the grading time simulations are repeated for a time grading of 125 ms. In table 6 the new settings of the protection schemes are shown.

Table 6: Protection settings, reduced grading

\begin{tabular}{|c|c|c|c|c|c|}
\hline & CT- & \multicolumn{2}{|c|}{ Definite } & \multicolumn{2}{c|}{ Inverse } \\
\cline { 3 - 6 } & Ratio & $\mathrm{I} \gg>$ & $\mathrm{t} \gg>$ & $\mathrm{T}_{\mathrm{p}}$ & $\mathrm{I}_{\mathrm{p}}$ \\
\hline Sub 1 & $750 / 5 \mathrm{~A}$ & $4500 \mathrm{~A}$ & $0,6 \mathrm{~s}$ & 0.2 & 7.1 \\
\hline Sub 2 & $750 / 5 \mathrm{~A}$ & $4500 \mathrm{~A}$ & $0,475 \mathrm{~s}$ & 0.17 & 7.1 \\
\hline Sub 3 & $750 / 5 \mathrm{~A}$ & $4500 \mathrm{~A}$ & $0,350 \mathrm{~s}$ & 0.13 & 7.1 \\
\hline Sub 4 & $750 / 5 \mathrm{~A}$ & $4500 \mathrm{~A}$ & $0,225 \mathrm{~s}$ & 0.09 & 7.1 \\
\hline Sub 5 & $750 / 5 \mathrm{~A}$ & $4500 \mathrm{~A}$ & $0,1 \mathrm{~s}$ & 0.05 & 7.1 \\
\hline
\end{tabular}

For the new settings the average clearing time is also assessed. The average clearing time is $0.35 \mathrm{~s}$ for the definite over-current protection and $0.38 \mathrm{~s}$ for the inverse over-current protection.

\section{EFFECT OF GRID PROTECTION ON STABILITY OF DG-UNITS}

In this section the effect of grid protection on stability of DG units is discussed. In the previous section it is already mentioned that the average clearing time can be reduced by reducing the time grading, however the average clearing time still exceeds the CCT of the DG units. The definite and the inverse over-current protection schemes are evaluated with the aid of the complete test grid. The disturbances are put in feeder 1 . As mentioned earlier, with the current time grading $(300 \mathrm{~ms})$ stable operation of all DG-units is only guaranteed when the disturbance occurs between substation 4 and 5 . When applying the reduced time grading $(125 \mathrm{~ms})$ stable operation of the DG- 
units is guaranteed for disturbances between substation 3 and 5. This only holds for the definite over-current protection scheme. In figure 6 the rotor angles of all DGunits are shown during short circuit of sub 4 and after fault clearing.

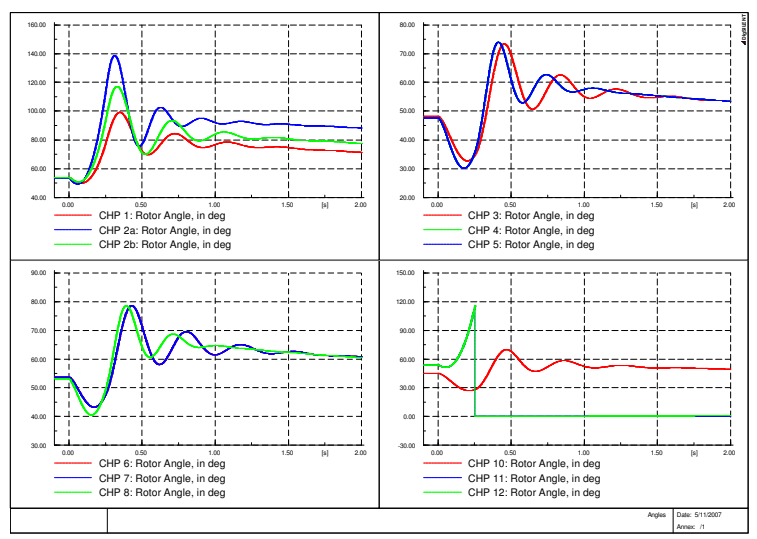

Figure 6 Dynamic response DG-units to a short circuit of substation 4 for a definite over-current protection scheme

In figure 6 the rotor angle of CHP11 and 12 gets zero which means that the fault is cleared by the protection relay. Rotor oscillations of all other DG-units are damped to a stable value. The simulation is also performed for inverse over-current protection scheme. The results are depicted in figure 7.

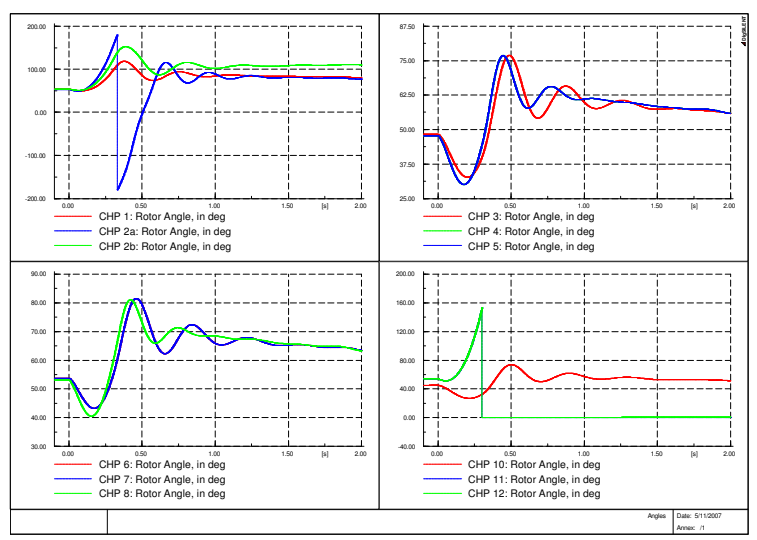

Figure 7 Dynamic response DG-units to a short circuit of substation 4 for an inverse over-current protection scheme

In figure 7 the rotor angle of CHP11 and 12 also gets zero however, the fault is cleared in $300 \mathrm{~ms}$. This results in a larger increment of the rotor angle of all other DG-units and even instability of CHP2a. For all other disturbances on feeder 1 both protection schemes shows instability of DG-units.

Stable operation can be guaranteed by clearing the fault within $200 \mathrm{~ms}$. It is shown that this cannot be reached with the proposed protection schemes. Due to the large difference of the nominal current of the feeder and the nominal current of the generators, the pickup currents of the applied protection relays also have a large difference (see figure 3). Without the loss of selectivity the clearing time of the current protection scheme can be set to $100 \mathrm{~ms}$. A sacrifice is made to clear the complete feeder with loss of all the generators connected to the feeder however all remaining generators stay in stable operation. In figure 8 the results of the simulation of this protection setting is shown. A sacrifice is made to clear the complete feeder with loss of all the generators connected to the feeder however all remaining generators stay in stable operation.

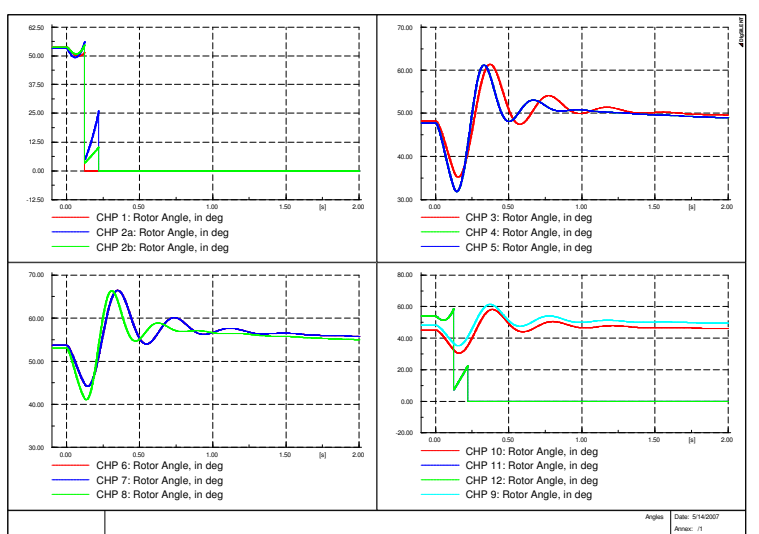

Figure 8 Dynamic response DG-units to a short circuit of substation 4 for a definite over-current protection scheme of outgoing feeder

\section{CONCLUSIONS}

In this paper the effect of grid protection on stability of DG-units is studied. Evaluation of the current protection scheme shows that all DG-units are disconnected by the under-voltage protection before the fault is cleared by the grid protection. When the under-voltage protection is omitted the current protection scheme shows that all DGunits become unstable and are disconnected by its overcurrent relay. In this situation the under-voltage protection is essential and prevents any damage to the DG-units.

To increase the availability of the DG-units and reduce the fault clearing time, definite over-current protection and inverse over-current protection schemes are considered. The protection schemes are evaluated for classical time grading $(300 \mathrm{~ms})$ and reduced time grading $(125 \mathrm{~ms})$. Both protection schemes lead to approximately the same average clearing time. Hence for short feeders inverse over-current protection schemes yield no extra benefit in comparison to definite over-current protection schemes.

In order to guarantee stable operation for most part of the test grid, a compromise has to be made. Accepting loss of a complete feeder allows a simple definite over-current protection on the outgoing feeder in the substation. In combination with the under-voltage protection this is an adequate way of protecting MV-grids with short feeder length and preventing damage to the DG-units.

\section{REFERENCES}

[1] 1547 IEEE Standard for Interconnecting Distributed Resources with Electric Power Systems, IEEE, New York, USA July, 2003

[2] Gatta, F.M., et al, Modeling and Computer Simulation of Dispersed Generation in Distribution Networks. Measures to prevent disconnection during system disturbances, IEEE Bologna PowerTech Conference, June 23-26, Bologna, Italy, 2003

[3] Buchholz, B.M., et al, Dynamic Simulation of Renewable Energy Sources and Requirements of Fault Ride Through Behavior, IEEE, 2006 
[4] Kundur, P., Power System Stability and Control, McGraw Hill, New York, USA, 1993

[5] Grainger, J.J., Stevenson, W.D., Power System Analysis, McGraw Hill, Inc, 1994

[6] Jäger, J., Keil, T., Shang, L., Krebs, R., New Protection Coordination Method in the Presence of Distributed Generation, Siemens AG, Germany, 2004

[7] SIEMENS instruction manual, Numerical Time Overcurrent Protection and thermal overload relay with auto-reclosure option SIPROTEC 7SJ600, v3.1, Siemens AG, 2001

[8] Gers, J.M., Holms, E.J., Protection of Electricity Distribution Networks, IEE, London, UK, 2004

\section{AUTHOR'S ADDRESS}

The first author can be contacted at

\section{ENECO NetBeheer}

Afdeling Assetmanagement

Rochussenstraat 200

3015 EK Rotterdam, The Netherlands ejcoster@ieee.org 